\title{
Doppler Frequency Parameters Estimation in the Fractional Fourier Transform Domain
}

\author{
$\mathrm{Li} \mathrm{Li} i^{1, a *}$ \\ ${ }^{1}$ College of Information Engineering, Dalian University, Dalian 116622, China \\ affsimple@163.com
}

\begin{abstract}
Keywords: Fractional Fourier transform; Fractional correlation; Doppler frequency; Parameter estimation
\end{abstract}

\begin{abstract}
A new method based on fractional correlation theory for estimating the Doppler parameters of the echoes signal is presented. Firstly, a signal array model is constructed with time-variant Doppler frequency. Secondly, one of the Doppler frequency parameters, initial Doppler frequency, is estimated by energy concentrated of multipath component based on fractional correlation transform. Then, an order-reduced signal is reconstructed combining the Doppler with the prior knowledge of the transmitted signal, and the echoes signal is converted to many single-frequency signals. Finally, the multipath time delay is obtained by the dechirping method. Simulation results are given in this paper and the results show that the new method is valid.
\end{abstract}

\section{Introduction}

The fractional Fourier transform (FRFT), which is a generalization of the classical Fourier transform, was introduced a number of years ago in the mathematics literature but appears to have remained largely unknown to the signal processing community, to which it may, however, be potentially useful. The FRFT depends on a parameter and can be interpreted as a rotation by an angle in the time-frequency plane.

Target parameter estimation is an important aspect in radar signal processing. Most existing algorithms present that Doppler frequency is time-invariant as shown in [1-3]. In fact, for a moving target, its echo often contains a time-varying Doppler frequency. Therefore, the received signals contain time-variant Doppler frequency due to the three dimension motion state of these targets. In this case, these existing methods can not effectively solve this problem and provide an optimal solution [5-10]. To overcome these drawbacks, this paper presents a novel method in the fractional Fourier transform, to estimate parameters based on fractional correlation.

The paper is organized as follows. Section 2 proposes a signal array model with time-variant Doppler frequency. In section 3, theory of Parameter Estimation based on the fractional correlation is presented. In section 4, Doppler frequencies Parameter are estimated based on Fractional Correlation. In section 5, the performance of the proposed parameter estimation algorithm is studied through extensive numerical simulations. Finally, conclusions are drawn in section 6.

\section{The Signal Model}

In radar or a sonar system, the received signal, in comparision with the transmitted waveform, often contains Doppler frequency. We assume that the transmit antennas emit orthogonal waveforms $x(t)$. Let $x(t)$ be the transmitted signal having a constant propagation velocity. The received signals contain Doppler frequency with time-variant. In general, the echo signal $y(t)$ can be expressed as

$$
y(t)=\sum_{l=1}^{L} \sigma_{l} x(t) \exp \left(j 2 \pi\left(f_{l} t+\mu_{l} t^{2} / 2\right)\right)+w(t)
$$


where $\sigma_{l}$ denotes the radar cross-section. $f_{l}$ and $\mu_{l}$ denote the initial Doppler frequency (IDF) and Doppler frequency rate (DFR). $L$ is the number of multiple paths. The noise $w(t)$ is assumed to be independent, zero-mean Gaussian white noise.

\section{Theory of Parameter Estimation Based on the Fractional Correlation}

FRFT. In recent years, the FRFT has drawn considerable attention in many areas such as quantum physics, optical systems and signal processing. The FRFT is a generalization of the FT, and can be interpreted as a rotation of the signal to any angles in the time-frequency plane ${ }^{[4]}$. The continuous FRFT of a signal $f(t)$ with angle $\alpha$ is defined as

$$
F(\alpha, m)=F^{\alpha}[f(t)](m)=\int_{-\infty}^{+\infty} f(t) K_{\alpha}(t, m) d t
$$

where $F^{\alpha}$ denotes the FRFT operator and $K_{\alpha}(t, m)$ is the kernel function of the fractional Fourier transform. $K_{\alpha}(t, m)$ can be expressed as

$$
K_{\alpha}(t, m)= \begin{cases}\sqrt{(1-j \cot \alpha)} \exp \left(j \pi\left(t^{2} \cot \alpha-2 m t \csc \alpha+m^{2} \cot \alpha\right)\right), \alpha \neq n \pi \\ \delta(t-m), & \alpha=2 n \pi \\ \delta(t+m), & \alpha=(2 n+1) \pi\end{cases}
$$

where $\alpha$ and $m$ are the rotation angle and the frequency in FRFT domain, respectively.

Fractional Correlation Function. Correlation function is fundamental tools in random signal analysis and processing [7]. Since the FRFT generalizes the FT into arbitrary angle of the time-frequency plane, it is worthwhile to consider the fractional counterpart of the conventional correlation function. They are based on the fractional Fourier transform and are thus coined fractional correlation function. Next, we give the definitions of the fractional correlation proposed.

Assume that the signal $s(t)$ is modeled as

$$
s(t)=b_{0} \exp \left(j 2 \pi\left(a_{1} t+a_{2} t^{2} / 2\right)\right)
$$

where $b_{0}$ is the signal amplitude, $a_{1}$ is the initial frequency and $a_{2}$ is frequency rate.

The fractional correlation function $\hat{R}_{s s}(\alpha, \tau)$ in the FRFT domain of the signal $s(t)$ is defined by

$$
\hat{R}_{s s}(\alpha, \tau)=\lim _{T \rightarrow \infty} \frac{1}{2 T} \int_{-T}^{+T} R_{s s}(t+\tau, t) \exp (j t \tau \cot \alpha) d t
$$

where $R_{s s}(t+\tau, t)$ is the correlation function of the signal $s(t), \tau$ denotes the time delay, and $\rho$ is the rotation angle in FRFT domain.

\section{Joint Doppler Frequency Parameter Estimation Based on Fractional Correlation}

According to (1) and (6), the fractional correlation function of $y(t)$ can be written as

$$
\hat{R}_{y y}(\tau)=\lim _{T \rightarrow \infty} \frac{1}{2 T} \int_{-T}^{+T} R_{y y}(t+\tau, t) \exp (j t \tau \cot \alpha) d t
$$

where $R_{y y}(t+\tau, t)=E\{y(t+\tau)[y(t)]\}$.

When $\cot \alpha_{l}=-2 \pi \mu_{l}, \hat{R}_{y y}(\tau)$ has the best energy-concentrated property. Therefore, we can obtain the following expression as

$$
\left\{\begin{array}{l}
\hat{\mu}_{l}=-\cot \alpha_{l} /(2 \pi) \\
\hat{f}_{l}=\arg \max _{f}\left\{\tilde{R}_{y_{1}}(u)\right\}
\end{array}\right.
$$


where $\tilde{R}_{y_{1}}(u)$ is the Fourier transform of $R_{y_{1}}(t)$, and $R_{y_{1}}(t)$ is the autocorrelation function of $y_{1}(t)=y(t) \cdot \exp \left(-\mathrm{j} 2 \pi\left(\hat{\mu}_{l} / 2\right) t^{2}\right)$.

\section{Simulation Results}

The number of target is $L=2$, Doppler frequency parameters are $f_{1}=8, \mu_{1}=0.5, f_{2}=12, \mu_{2}=0.9$, respectively. The sampling frequency $f_{s}$ is $100 \mathrm{~Hz}$ and the number of snapshots is 800 . The number of Monte Carlo iterations is 500 in all simulations. We use the root-mean-square error (RMSE) [8] of the $l$ th target $R M S E_{\xi_{l}}=\sqrt{\frac{1}{K} \sum_{k=1}^{k=K}\left(\hat{\xi}_{l k}-\xi_{l}\right)^{2}}$ as performance measurement, where $K$ is the Monte-Carlo trial number, $\hat{\xi}_{l k}$ is the estimate of the $k$ th experiment and $\xi_{l}$ is the true value, and $\xi_{l}$ denotes $f_{l}$ and $\mu_{l}$, respectively.

In this simulation, we consider the influence of the SNR to the performance of the proposed method. Fig.1 shows the RMSE of Doppler frequency parameters estimation of the proposed method versus SNR. Fig. 2 shows the RMSE of Doppler estimation of the proposed method and the PARAFAC method [7]. From Fig. 1, we see when SNR is higher than -5dB, the RMSE curves are flat. From Fig. 2 , the performance of the proposed method is significantly better than that of the PARAFAC method.

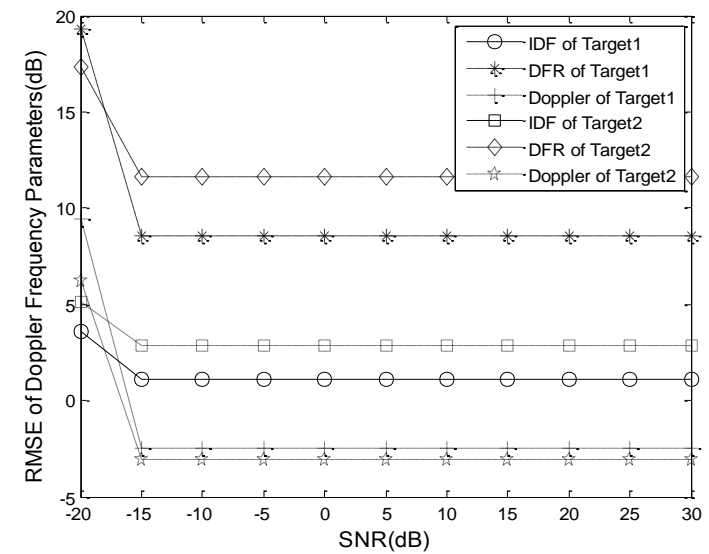

Figure 1. RMSE of Doppler parameters estimation versus SNR

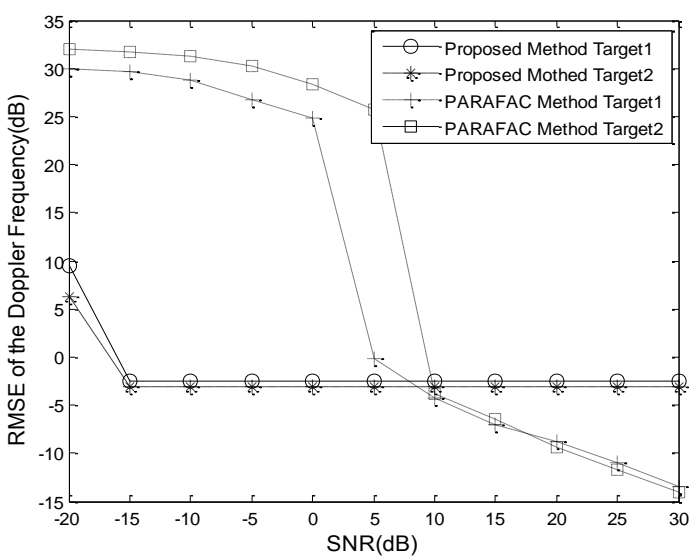

Figure 2. RMSE of both methods versus SNR

For a given delay $\tau$, instantaneous autocorrelation function of the cubic phase signal is a linear frequency modulation signal, and the linear frequency modulation signal has the characteristic energy-concentrated in FRFT domain while noise does not have such characteristic, so Doppler 
frequency parameters can be estimated by searching peak of the fractional ambiguity function. The received signal has a low SNR in bistatic MIMO radar system, so the proposed method is more suitable for this application. It is important to find better peak searching method so as to improve the estimation accuracy.

\section{Conclusion}

In this paper, we proposed a novel method for estimating unknown targets parameters in fractional Fourier transform domain. Firstly, we present a signal model with time-variant Doppler frquency. Secondly, Doppler parameters are estimated by searching the peak of fractional correlation. Simulation results demonstrate that the proposed method still has good performance when poor SNR condition exists.

\section{Acknowledgments}

This work was partly supported by the National Science Foundation of China under Grants 61401055 .

\section{References}

[1] C.Y. Chen and P.V. Palghat, MIMO radar space-time adaptive processing using prolate spheroidal wave functions, IEEE Transactions on Signal Processing, 56(2008) 623-635.

[2] C.C. Li, et al, An ESPRIT-like algorithm for coherent DOA estimation based on data matrix decomposition in MIMO radar, Signal Processing, 91(2011) 1803-1811.

[3] M.L. Bencheikh, Y.D. Wang and H.Y. He, Polynomial root finding technique for joint DOA DOD estimation in bistatic MIMO radar, Signal Processing, 89(2010) 2723-2730.

[4] R. Tao, F. Zhang, Y. Wang, Fractional Power Spectrum, IEEE Trans. Signal Processing, 56(2008) 4199-4206.

[5] H. Lu, et al, A novel method for target localization and doppler frequency estimation in bistatic MIMO radar, Journal of Electronics and Information Technology, 32(2010) 2167- 2171.

[6] X.F. Zhang, et al, Direction of departure (DOD) and direction of arrival (DOA) estimation in MIMO radar with reduced-dimension MUSIC, IEEE Communications Letters, 14(2010) 1161-1163.

[7] J.Y. Zhang, Z.D. Zheng, and X.B. Li, An algorithm for DOD-DOA and Doppler frequency jointly estimating of bistatic MIMO radar, Journal of Electronics and Information Technology, 32(2010): 1843-1848.

[8] C.L.Nikias and S. Min, Signal processing with alpha-stable distributions and applications. Wiley-Interscience, 1995.

[9] L.B. Almeida, The fractional Fourier Transform and Time-Frequency Representations, IEEE Trans. Signal Processing, 42(1994):3084-3091.

[10] J.T. Wang, et al. Subspace-based DOA Estimation in Impulsive Noise Environments for MIMO Radars, Journal of Astronautics, 30(2009) 1653-1657. 